\title{
Capacitação em serviço de nutrição e dietética: implantação do protocolo de identificação correta do paciente no fornecimento de dietas hospitalares
}

Training in nutrition and dietetics service: implantation of the correct patient identification protocol in hospital diets supply

Capacitación en servicio de nutrición y dietética: implantación del protocolo de identificación correcta del paciente en el suministro de dietas hospitalarias

Thaís de Oliveira Carvalho Granado Santos ${ }^{1 *}$, Xaene Maria Fernandes Duarte Mendonça ${ }^{2}$, Pilar Maria de Oliveira Moraes ${ }^{3}$, Marina Goreth Silva de Campos$^{2}$, Jamilie Suelen dos Prazeres Campos ${ }^{1}$, Lizomar de Jesus Maués Pereira Moia², Naiza Nayla Bandeira de Sá2, Vera Lúcia de Azevedo Lima $^{2}$, Silvia Ferreira Nunes ${ }^{3}$, Camila de Cássia da Silva de França ${ }^{3}$, Paula Regina Ferreira Lemos ${ }^{3}$, Ana Carla Pinto da Silva ${ }^{4}$, Rahilda Conceição Ferreira Brito Tuma ${ }^{4}$.

\section{RESUMO}

Objetivo: Descrever a capacitação de colaboradores de um Serviço de Nutrição e Dietética sobre segurança do paciente e identificação correta do paciente no fornecimento de dietas hospitalares. Relato de Experiência: Estudo descritivo tipo relato de experiência, elaborado a partir da capacitação realizada com os colaboradores do Serviço de Nutrição e Dietética de um hospital público em Belém-PA. A atividade incluiu exposição de vídeo, palestra expositiva, apresentação e avaliação de material educativo. Participaram 22 colaboradores que atuam como cozinheiros, copeiros, despenseiros e serviços gerais, os quais demonstraram surpresa com os eventos adversos que poderiam ser evitados pela adoção de protocolos enfocando a segurança do paciente e perceberam que os colaboradores do Serviço de Nutrição e Dietética atuam na assistência ao paciente e devem adotar as orientações para a melhoria do cuidado. Alguns desconheciam que a identificação correta do paciente pode minimizar riscos relacionados à assistência em saúde. Os colaboradores avaliaram o conteúdo da cartilha como adequado e consideraram a capacitação importante. Considerações Finais: A capacitação é uma ferramenta para a implantação de um novo processo de trabalho, sendo relevante envolver e valorizar a atuação dos profissionais no serviço para que atuem proporcionando segurança ao paciente e a qualidade do cuidado.

Palavras-Chave: Segurança do Paciente, Capacitação em Serviço, Serviço Hospitalar de Nutrição.

\footnotetext{
ABSTRACT

${ }^{1}$ Hospital Ophir Loyola (HOL), Belém-PA. *E-mail: thaissoliveira@yahoo.com.br

2 Universidade Federal do Pará (UFPA), Belém-PA.

${ }^{3}$ Fundação Santa Casa de Misericórdia do Pará (FSCMP), Belém-PA.

4 Secretaria de Estado de Saúde Pública (SESPA), Belém-PA.
}

Objective: To describe the training of the Nutrition and Dietetic Service team on patient safety and correct patient identification in the provision of hospital diets. Experience Report: A descriptive, experience-based study, based on in-service training with Nutrition and Dietetics Service employees of a public hospital in BelémPA. The activity included video, dialogued exhibition, and presentation and evaluation of educational material. Twenty-two employees who work as cooks, cupbearers, stewards and general services, which showed 
surprised at the adverse events that could be avoided by the adoption of protocols focusing on patient safety and perceived that Nutrition and Dietetics Services act in the patient care and should adopt guidelines for improving care. Some were unaware that the correct patient identification can minimize risks related to health care. The employees rated the educational material as appropriate and considered the training an important action. Final Comments: Inservice training is a tool for the implementation of a new work process, and it is important to involve and value the professionals' performance in the service so that they act to provide patient safety.

Key words: Patient Safety, Inservice Training, Hospital Food Service.

\section{RESUMEN}

Objetivo: Describir la capacitación del equipo de un Servicio de Nutrición y Dietética sobre seguridad del paciente e identificación correcta del paciente en el suministro de dietas hospitalarias. Relato de experiencia: Estudio descriptivo tipo relato de experiencia, elaborado a partir de la capacitación realizada con los colaboradores del Servicio de Nutrición y Dietética de un hospital público en Belém-PA. La actividad incluyó exposición de vídeo, exposición dialogada, presentación y evaluación de material educativo. Participaron 22 colaboradores que actúan como cocineros, copero, despensas y servicios generales, los cuales demostraron sorpresa con los eventos adversos que podrían ser evitados por la adopción de protocolos enfocando la seguridad del paciente y percibieron que los colaboradores del Servicio de Nutrición y Dietética actúan en la asistencia al paciente y deben adoptar las orientaciones para la mejora del cuidado. Algunos desconocían que la identificación correcta del paciente puede minimizar los riesgos relacionados con la asistencia en salud. Los colaboradores evaluaron el contenido de la cartilla como adecuado y consideraron la capacitación importante. Consideraciones Finales: La capacitación es una herramienta para la implantación de un nuevo proceso de trabajo, siendo importante involucrar y valorar la actuación de los profesionales en el servicio para que actúen proporcionando seguridad al paciente y calidad del cuidado.

Palabras clave: Seguridad del Paciente, Capacitación en Servicio, Servicio de Alimentación en Hospital.

\section{INTRODUÇÃO}

A Segurança do Paciente e a Qualidade do Cuidado passaram a mobilizar o mundo a partir do relatório lançado em 1999 pelo Institute of Medicine (IOM), "To Error is Human - Building a safer health system", no qual foram divulgados o grande número de eventos adversos relacionados à assistência à saúde, o que levou as organizações de saúde a estabelecer estratégias e direcionar ações visando a melhoria da qualidade da assistência prestada, a segurança da instituição e do colaborador envolvido (BRASIL, 2013a).

Entre as medidas adotadas foi criado em 2004 pela Organização Mundial da Saúde (OMS) o Programa Segurança do Paciente (PSP) com objetivo de coordenar, disseminar e acelerar as melhorias relacionada à segurança do paciente e o incentivo à adoção das Metas Internacionais de Segurança do Paciente (MISP) definidas pela OMS juntamente com a Joint Commission International (JCI) (BRASIL, 2013a).

O Brasil aderiu às estratégias globais e em 2013 estabeleceu o Programa Nacional de Segurança do Paciente (PNSP) com objetivo de contribuir para a qualificação do cuidado em saúde em todos os estabelecimentos do território nacional e que estabelece entre as estratégias de implementação, a elaboração e apoio à implementação de protocolos e manuais de segurança do paciente; a capacitação de profissionais e equipes de saúde em segurança do paciente e a promoção da cultura de segurança com ênfase no aprendizado e aprimoramento organizacional, engajamento dos profissionais e dos pacientes na prevenção de incidentes, com ênfase em sistemas seguros (BRASIL, 2013b).

Dentre os diversos cuidados que podem ser ofertados ao paciente atendido pelos estabelecimentos de saúde, Holmes B et al. (2010), destacam a assistência nutricional como parte essencial do cuidado ao paciente, e afirmam que um cuidado não apropriado ou ausente pode ser a causa de problemas de segurança 
em alguns pacientes. Segundo os autores, em estudo feito acerca dos incidentes de segurança registrados na Agência Nacional de Segurança do Paciente do Reino Unido, os incidentes de segurança relacionados à nutrição tinham em comum, temas emergentes, tais como: a comunicação deficiente entre setores e equipes, falhas na identificação das necessidades nutricionais dos pacientes, equipe em número insuficiente e pouco qualificada para fornecer cuidados nutricionais satisfatórios. Nieuwoudt $C$ (2014), buscou uma conexão entre a nutrição e a segurança do paciente e apontou a identificação do paciente como uma prática de cuidado com potencial para causar incidentes de segurança relacionados à nutrição.

Assim, os Serviços de Nutrição e Dietética (SND), também responsáveis pela assistência ao paciente, devem participar do processo de implantação de ferramentas que promovam a segurança do paciente e a qualidade do cuidado em saúde, sendo fundamental capacitar a equipe por meio da educação permanente em saúde, estratégia utilizada para transformar práticas profissionais e da organização do trabalho (FALKENBERG MB, et al., 2014).

A implantação de novas rotinas e protocolos para aumentar a segurança dos pacientes internados exige o desenvolvimento de estratégias de apoio educacional e ações de acompanhamento gerencial que sustentem práticas seguras e baseadas em evidências (HEMESATH MP, et al., 2015).

A utilização de tecnologias educativas construídas com conteúdo, linguagem e ilustrações adequadas, objetivando sempre o aprendizado e realizando intervenções educativas junto aos profissionais pode ser uma importante ferramenta nesse processo.

Diante do exposto, o objetivo do presente estudo foi descrever a capacitação de colaboradores de um Serviço de Nutrição e Dietética sobre segurança do paciente e a identificação correta do paciente no fornecimento de dietas hospitalares.

\section{MÉTODOS}

Trata-se de um estudo descritivo, do tipo relato de experiência, elaborado a partir do processo de capacitação em serviço realizado junto aos colaboradores do Serviço de Nutrição e Dietética (SND) de um hospital público de referência em oncologia no município de Belém, estado do Pará como etapa para a implantação do Protocolo de Identificação do Paciente no fornecimento das dietas hospitalares.

A capacitação foi realizada no dia 03 de setembro de 2018 nos turnos manhã e tarde e contou com a exibição de um vídeo educativo sobre o tema segurança do paciente, seguido de uma exposição dialogada e da apresentação de uma Cartilha sobre a Segurança do Paciente no Serviço de Nutrição Hospitalar, elaborada a partir dos tópicos "Qualidade e Segurança do Paciente", "Identificação correta do Paciente" e "Fluxograma do Atendimento e Identificação do Paciente no serviço", e da avaliação do material educativo pela equipe de colaboradores.

Este estudo faz parte da Dissertação "Segurança do Paciente na Identificação de Dietas Hospitalares", apresentada ao Programa de Pós-Graduação em Gestão e Saúde na Amazônia, a qual seguiu todas as normas de pesquisa envolvendo seres humanos e foi aprovada pelos Comitês de Ética em Pesquisa da Fundação Santa Casa de Misericórdia do Pará (FSCMP) e Hospital Ophir Loyola (HOL), sob parecer $n^{\circ}$ 2.632.514 e $\mathrm{n}^{\circ} 2.6999 .403$, respectivamente.

\section{RELATO DE EXPERIÊNCIA}

Para difundir as novas práticas e rotinas a serem adotadas no serviço foi planejada a realização de atividade educativa com os colaboradores do Serviço de Nutrição e Dietética (SND) de um hospital público em Belém-PA. A capacitação foi realizada no dia 03 de setembro de 2018 em 02 (dois) turnos para garantir que houvesse a participação de um maior número de colaboradores, os quais foram convidados a participar da atividade educativa de forma voluntária, respeitando os preceitos da ética em pesquisa. Um total de 26 (vinte e seis) colaboradores de ambos os sexos, na faixa etária de 26 a 64 anos, participaram da capacitação 
nos dois momentos, os colaboradores do cargo auxiliar operacional, os quais atuam nas funções de copeiros, cozinheiros, despenseiros e serviços gerais.

A atividade teve início com uma breve explicação sobre o tema para que os mesmos pudessem compreender determinados conceitos antes de iniciar a segunda etapa. Em seguida, os colaboradores foram convidados a assistir um vídeo educativo sobre o "Segurança do paciente", no qual é apresentado o caso de uma paciente oncológica, que recebeu um cuidado inseguro, o que ocasionou um desfecho trágico para a paciente. No vídeo, verifica-se que uma série de protocolos e barreiras, elaborados para garantir segurança ao paciente, à equipe e à instituição, não foram seguidos, o que causou um grave incidente à paciente. $A$ partir da situação exposta no vídeo, foram levantadas algumas questões que foram discutidas com o grupo participante.

Após essa etapa, os colaboradores assistiram a uma expositiva intitulada "Segurança do Paciente no Serviço de Nutrição Hospitalar", em que foram abordadas informações sobre o histórico da segurança do paciente, conceitos sobre segurança do paciente e qualidade do cuidado, Metas Internacionais de Segurança do Paciente, Programa Nacional de Segurança do Paciente, Protocolo de Identificação correta do paciente, bem como a relação entre segurança do paciente e o serviço de nutrição. A apresentação iniciou com a problematização acerca do que as falhas na identificação do paciente podem causar, alertando aos profissionais que a utilização de etiquetas de identificação nas dietas e a simples conferência dos identificadores do paciente podem evitar uma série de eventos adversos (Figura 1).

Figura 1 - Palestra expositiva sobre segurança do paciente para colaboradores do Serviço de Nutrição e Dietética de um hospital público em Belém-PA, 2018.

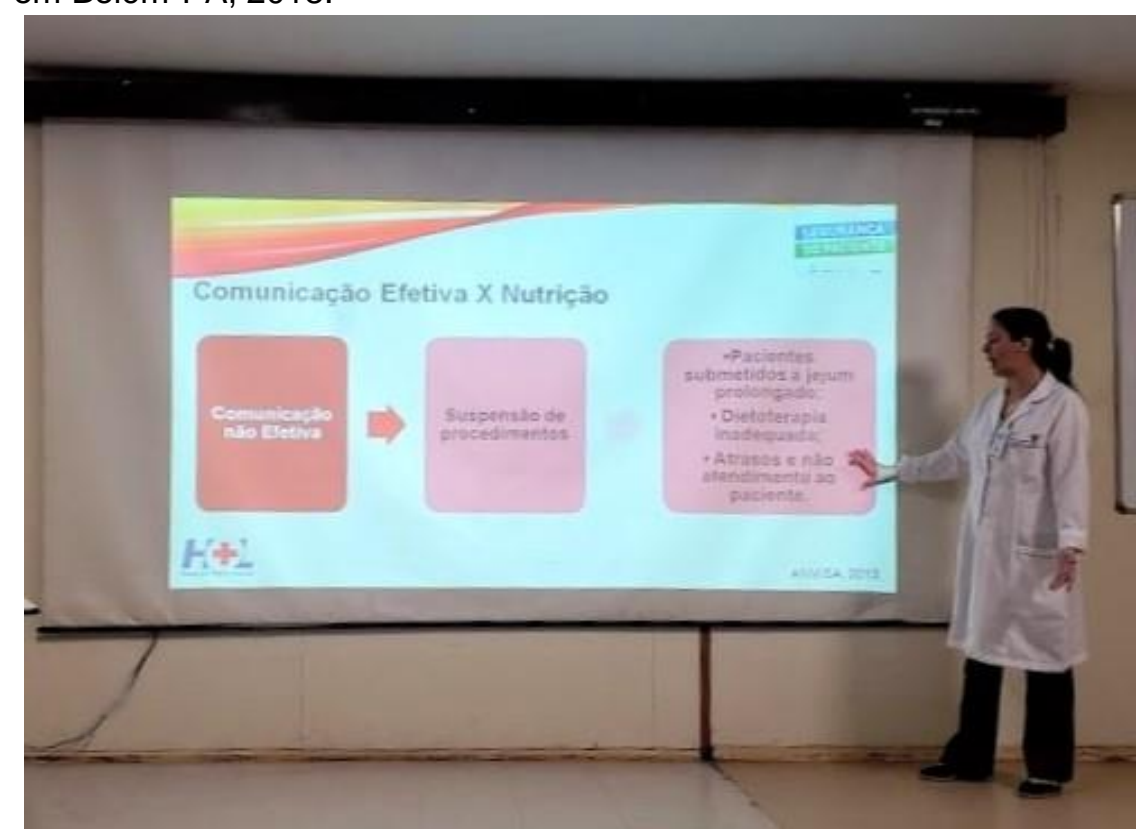

Fonte: Dados da Pesquisa, 2018.

Os colaboradores demonstraram surpresa quando verificaram a quantidade de eventos adversos relacionados ao cuidado em saúde e que podem ser evitados pela adoção de regras e protocolos de assistência visando garantir a segurança do paciente.

Também tiveram a oportunidade de perceber que o SND, como parte do estabelecimento de saúde, também é importante na assistência ao paciente e deve adotar as orientações preconizadas para a melhoria do cuidado em saúde. Alguns desconheciam que é necessário identificar corretamente o paciente e que esse procedimento é capaz de minimizar riscos relacionados à assistência em saúde. 
A última etapa da atividade consistiu na apresentação e distribuição de uma cartilha sobre a segurança do paciente no Serviço de Nutrição Hospitalar (SANTOS TOCG, et al., 2018), a qual foi apresentada passo a passo para que os participantes pudessem compreender e tirar dúvidas sobre 0 assunto. $O$ material apresentou resumidamente o tema segurança do paciente e as metas instituídas pela OMS (Organização Mundial da Saúde) e JCl (Joint Commission International), além de expor a importância da identificação correta do paciente em todos os cuidados direcionados ao paciente inclusive no fornecimento de dietas e a necessidade de conferir os dados de identificação ao fornecer as dietas aos pacientes (Figura 2).

Figura 2 - Recorte da Cartilha sobre Segurança do Paciente no Serviço de Nutrição Hospitalar, 2018.

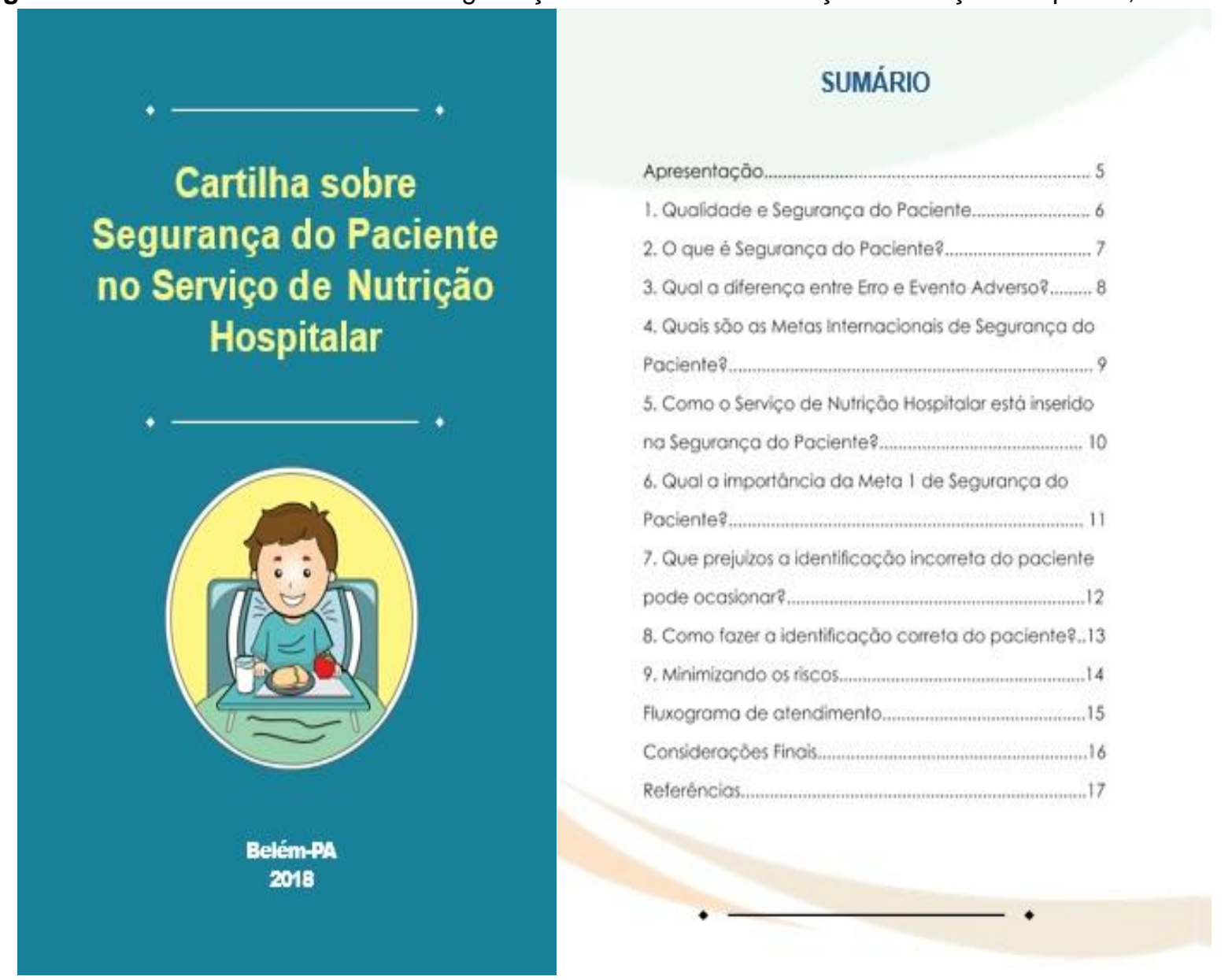

Fonte: Dados da Pesquisa, 2018.

Ao final, os colaboradores da DND/HOL (Divisão de Nutrição e Dietética/Hospital Ophir Loyola) participaram de uma pesquisa opinativa, em que puderam avaliar o conteúdo da cartilha, as cores e as imagens utilizadas, sendo que a maioria dos colaboradores considerou adequado e ainda expressaram comentários como "foi muito bom o curso", "foi tudo ótimo", "gostei muito do evento, que bom abordar esse assunto", "eu achei muito importante tudo".

\section{DISCUSSÃO}

No serviço de saúde no qual o treinamento foi realizado, os identificadores utilizados são o nome completo do paciente e sua data de nascimento. Cada paciente recebe uma pulseira de identificação com tais dados no momento da internação e conforme preconiza o PSP, a cada procedimento ou cuidado realizado, incluindo 
a administração de medicamentos, alimentos ou demais procedimentos, os dados do paciente são conferidos e checados pelo profissional de saúde.

Segundo a Agência Nacional de Vigilância Sanitária (ANVISA), no período de março de 2014 a dezembro de 2018, foram notificados mais de 272 mil incidentes em serviços prestadores de saúde no Brasil. Dentre os quais, as falhas na identificação do paciente e na administração de dietas compreendem aproximadamente 18 mil e 5 mil casos, respectivamente (BRASIL, 2019). Vale ressaltar que os dados costumam ser subestimados, haja vista, que diversos eventos não são notificados ou sequer percebidos pela equipe de saúde ou paciente/família/acompanhante.

A falha na identificação do paciente e/ou dietas é um fator que pode provocar incidentes em um serviço de saúde, porém, raramente é abordado no setor de nutrição hospitalar. Sendo assim, o treinamento permite que os colaboradores do SND conheçam e discutam sobre o tema segurança do paciente.

Embora todos os colaboradores do SND pudessem participar da capacitação, na prática, apenas o cargo de auxiliar operacional, frequentou, provavelmente porque estes se sentem diretamente implicados no processo, já que são os responsáveis diretos pelo fornecimento de dietas hospitalares, deixando-os passíveis de notificação e responsabilização no caso de falhas.

Desta forma, a adoção de estratégias de sensibilização e educação da equipe tem sido indicada como uma importante ferramenta, no processo de implantação da segurança do paciente, nos estabelecimentos de saúde, de modo que as novas práticas sejam implantadas de maneira colaborativa e construtiva (HEMESATH MP, et al., 2015). Para os autores, envolver os profissionais no processo é fundamental para alcançar a segurança e qualidade almejadas.

A OMS recomenda a educação para a segurança do paciente, inclusive sugerindo que o tema seja incluído nos desenhos curriculares dos cursos de saúde; e a educação permanente em saúde é uma das estratégias que pode ser usada nesse processo, sendo essencial para as transformações no trabalho e suas relações (WEGNER W, et al., 2016).

Relacionada à formação e desenvolvimento de profissionais para atuação na saúde, a educação permanente em saúde, enfoca na problematização do processo de trabalho e objetiva a transformação das práticas profissionais e da organização do trabalho, tendo como espaço o próprio local de trabalho e utilizando metodologias educativas que permitam a participação de todos (FALKENBERG MB, et al., 2014).

Uma das metodologias empregadas, a cartilha educativa, foi distribuída aos colaboradores, a qual teve como objetivo permitir que o processo de educação fosse contínuo, fornecendo constante informação para o leitor e auxiliando no momento do trabalho. Moreira MF et al. (2003) reúne em seu trabalho as principais características para a construção de um material educativo impresso, principalmente relacionado a área da saúde. Segundo os autores, é preferível a utilização de uma linguagem simples, para que a mensagem seja repassada de forma clara e convidativa. Na capacitação, os colaboradores demonstraram interesse na leitura da cartilha e elogiaram a produção, demonstrando que a mesma obedecia às características, propiciando maior entendimento do público.

Como método para educação continuada dos colaboradores, o treinamento, segundo o trabalho de revisão de Leandro AIP e Branco ES (2011), é essencial para conferir qualidade nas ações prestadas por um serviço de saúde, sendo visto como uma ferramenta importante para a atualização e melhoria do desempenho profissional.

No estudo de Wegner W et al. (2016), que objetivou conhecer as estratégias adotadas para a construção da cultura da segurança do paciente, na perspectiva dos profissionais da saúde, em um hospital universitário do Sul do Brasil, os participantes citaram o treinamento em serviço por meio de palestras, cursos e aulas teóricas como possibilidade educativa para apresentar a temática da segurança do paciente. Para os autores, a educação permanente em saúde incentiva a diversificação de estratégias para compartilhar conhecimentos 
e experiências do mundo do trabalho e a inclusão do tema segurança do paciente, por meio de rodas de conversa, provoca os profissionais da saúde para uma reflexão sobre as suas práticas de trabalho.

\section{CONSIDERAÇÕES FINAIS}

A capacitação em serviço é uma importante ferramenta para a implantação de um novo processo de trabalho em saúde, levando à transformação nas rotinas a partir da vivência no próprio serviço. Envolver os profissionais, escutar de forma ativa suas opiniões e experiências é uma forma de valorizar sua atuação no serviço, além de ser fundamental para que se sintam motivados e tenham clareza quanto as suas responsabilidades e papel desempenhado no serviço, para que assim atuem com mais atenção na qualidade de toda cadeia produtiva e possam proporcionar segurança ao paciente.

\section{AGRADECIMENTOS}

À Divisão de Nutrição e Dietética e a Diretoria do Hospital Ophir Loyola, que permitiram a realização do presente estudo e a Fundação Santa Casa de Misericórdia do Pará, em especial à equipe do Programa de Pós-Graduação em Gestão e Saúde na Amazônia, pelo apoio na sua realização.

\section{REFERÊNCIAS}

1. BRASIL. Ministério da Saúde (BR). Agência Nacional De Vigilância Sanitária. Relatório do Estados - Eventos Adversos.

2. BRASIL. Ministério da Saúde (BR). Agência Nacional de Vigilância Sanitária. Assistência Segura: Uma Reflexão Teórica Aplicada à Prática. Brasília (DF): 2013a.

3. Brasil. Ministério da Saúde. Portaria no 529, de 1 de abril de 2013. Institui o Programa Nacional de Segurança do Paciente (PNSP). Diário Oficial [da] República Federativa do Brasil. 2013 abr. 02; N. 62, Seção 1. p 43b.

4. FALKENBERG MB, et al. Educação em saúde e educação na saúde: conceitos e implicações para a saúde coletiva. Ciência Saúde Coletiva, 2014; 19(3): 847-852.

5. HEMESATH MP, et al. Estratégias educativas para melhorar a adesão à identificação do paciente. Revista Gaúcha de Enfermagem, 2015; 36(4): 43-8.

6. HOLMES B, et al. Nutrition-related patient safety incidents. Proceedings of the Nutrition Society, 69 (OCE7), E529, 2010.

7. LEANDRO AIP, BRANCO ES. Importância do treinamento e desenvolvimento nos serviços de saúde. RAHIS, 2011, (6): 64-69.

8. NIEUWOUDT C. Patient safety and nutrition: is there a connection? South African Journal of Clinical Nutrition, 2014; 27(3): 102-105.

9. MOREIRA MF, et al. Comunicação escrita: contribuição para a elaboração de material educativo em saúde. Revista Brasileira de Enfermagem, 2003, 56(2):184-188.

10. SANTOS TOCG, et al. Cartilha sobre segurança do paciente no serviço de nutrição hospitalar. Belém, PA, 2018. 18p. Disponível em: http://educapes.capes.gov.br/handle/capes/431488. Acesso em 14 abr. 2019.

11. WEGNER W, et al. Educação para cultura da segurança do paciente: Implicações para a formação profissional. Escola Anna Nery Revista de Enfermagem, 2016; 20(3). 